\title{
A Study on Application of Cyber Physical System (CPS) in Lime Production
}

\author{
Youli Zhou \\ Department of Bioinformatics Engineering, Yiyang Vocational \& technical College, Yiyang Hunan, \\ 41300, China
}

Key words: Plaster, Automation, The configuration system.

\begin{abstract}
Lime is important in the process of production of iron and steel materials, lime quality determines the quality of steel, lime production in China is much, but quality is not high, low efficiency, such as a direct result of the iron and steel production quality is not high. Automation so around this aspect research, in order to produce high quality white, produce high quality steel.
\end{abstract}

\section{Introduction and Scheme Design}

\section{Accepted Control System Scheme: CPS Automatic Control System}

For purpose of the control system bidding scheme, we adopt the CPS automatic control system with AHC2001 hybrid controller as the core that is manufactured by Azbil Corporation and widely used for real-time control and monitoring of a 200,000 T/Y lime production equipment.

(1) CPS system consists of control program assist-CPA; coordinating controller-AHC2001 hybrid multifunction controller.

Control program assist (CPA) refers to graphic man-machine user interface running on Microsoft Windows NT/2000 operating system platform, of which the software attributes can be classified into engineer and operator. The attributes can be customized.

(2) AHC2001 hybrid multifunction controller refers to high-precision controller developed by Azbil Corporation based on its longtime experience in the control field and with its controller technology that have been verified on spot.

(3) The print server supports network printer. Hence, line-at-a-time printer and color ink jet printer both can realize print sharing via the network printer server. Line-at-a-time printer is usually used for print alarm records, while color ink jet for printing statements and copied images.

\section{Scheme Description and CPS Configuration}

\section{Scheme Description:}

The system handles four processes, i.e. loading, preheating, slewing and cooling. According to the drawings you provided: the abovementioned four parts are independent from each other in respect of control, and also coordinate with each other to constitute a whole control system to carry out the whole production process from loading to cooling.

Based on the above information, we design a separate controller system for each of the abovementioned process, which are designed to realize data exchange via Ethernet. Between the host computer and all controllers, data exchange also is realized via Ethernet bus. The advantage of this is that the whole system follows a uniform data transmission protocol, so that data exchange is more convenient, flexible, transparent and faster. The connection diagram of each single system is as shown below: 


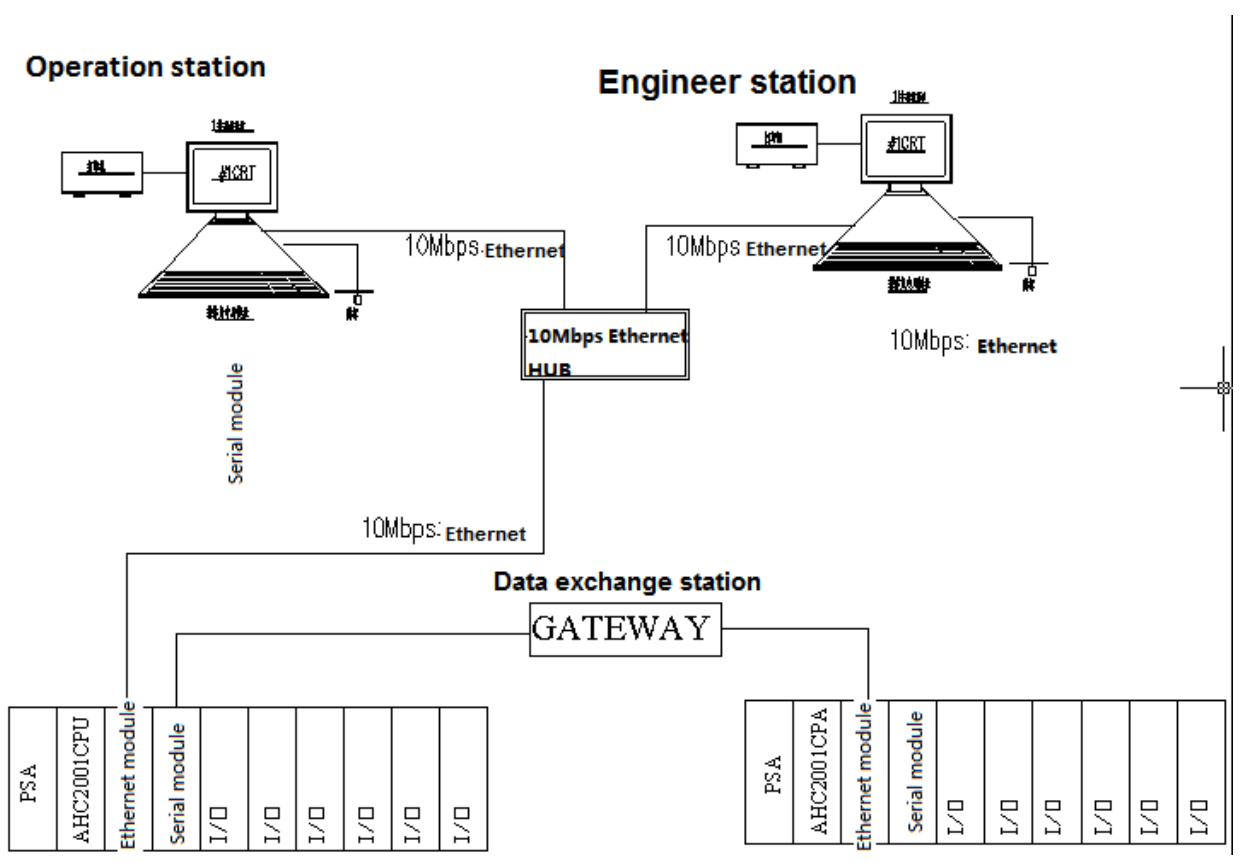

Specific configuration: 1 upper operation station, 1 engineer station. Engineer can use it for programming and configuration. The operation station is configured with operator attributor, which is for the use of operator.

CPS system allows connection with the third party control system via serial interface and Ethernet interface.

The print server supports network printer, so as to enable laser printer and color ink jet printer to realize network sharing as network printer, and visit client PC via the company' s information network to monitor historical information and batch processing.

\section{CPS Description}

The system has the features as below:

Open architecture:

For CPS, Microsoft Windows NT/2000 operating system is adopted. At the present stage, Windows NT/2000 is one of the operating systems of open architecture that are used most widely. It is convenient to connect P-LAN and a company LAN via TCP/IP protocol. The objects of other systems also can be switched in via OPC (OLE used for process control) or inset via DDE technology.

Easy to operate and maintain:

CPS is with a useful engineering tool developed by Azbil Corporation, which is not only used for the design of control strategy, but also for graphic and visual mode and creating engineering database/file for CPS system. SLPD-50 generates controller database that can be directly loaded in AHC2001 (coordinating controller).

The database created with SLPD-50 is easy to modify, update and extend in the same manner online in future.

Controller having been verified on spot:

AHC2001 hybrid controller is used as the CPS controller, which can provide powerful logic block based on Boolean operation, text language of ST structure easy to use, regulation control function, and regulation calculation block with hybrid point technology.

Hardware Components

CPA runs on personal computer: Pentium processor, 512MB SD RAM, CRT monitor: available sizes range from 17" to 21", with or without touch screen, mouse and QWERTY 101 keyboard.

One or two printers can be connected to CPA via the parallel or serial channel of personal computer. Color printer, laser printer and line printer all can be used here. 
Digital multipurpose compact disc (CD) is a movable backup medium, which can be used for storing the whole engineering database and storing historical data for reference and analysis in future.

Operation Security

CPS system distinguish operator from engineer via password, in which different persons have different operating authorizations.

Engineer' s Operating Station

Coordination monitor station: CPA supports two types of operating characteristics: operator characteristic and engineer characteristic (also called universal characteristic). No new software needs to be installed in any engineering environment. If an engineer changes some control structures via CRT, the change will come into force immediately, and can be stored into the controller online.

Operating Method

There are three operating media used for CPA, i.e. 101 QWERTY keyboard, mouse and touch screen.

In addition to mouse, operator also can realize operation via the touch screen of CRT. For group pictures and graphic pictures, operator can operate the set points, and control output via the up and down buttons on the screen of CRT.

Graphic Images

CPS system runs in Windows NT. Hence, bitmap units can be established. Moreover, the system has been installed with more than 60 graphic symbols. You can easily create new graphic symbols with the graphic editor. You can zoom in or out graphic symbols via the mouse. Every graphic symbol has its own attributes, such as color, size, use and function of defining users' statement. Among the standard graphic symbols, bar graph, tendency chart and component diagram are created with OCX programming technique. The graphic pictures can be embedded and connected with other Windows applications, such as objects created with Microsoft Excel and Word, without affecting the image display function. The image types are as follows:

Overview and Process Flow Diagram

The overview and process flow diagram shows the running state of devices, apparatuses and areas of the system, and the state, measured value, setting value, control mode (manual/automatic/cascade), and high or low level alarm and such information of procedure parameter variables, and can call out other images from the display block.

Rotary Monitoring Picture

The rotary monitoring pictures are used for display and control the running state and the sending of operating instructions of devices of the rotary furnace.

Trend Picture

Trend picture shows the trend of any data marker of high way, and the trend record curve of eight variables in the same coordinate axis, in which parametric variables, colors and time intervals are available for users to select. Besides, the data axis can be zoomed in at random.

\section{Alarm Screen}

The alarm screen displays all procedure parameter alarms and hardware breakdown alarms in progress, can sequence the alarms in reversed chronological order, and differentiates the priority level and state of alarms via different colors. Unidentified alarms are flashing.

Alarm contents cover:

- Alarm time

- Process variable name

- Process variable description

- Present value of process variable

- Setting value of alarm

- Engineering unit of process variable

- Priority level of alarm

Process Flow Diagram Screen 
The graphic picture shows the images of process units, process flow diagram, schematic diagrams of equipment, line diagram, etc. Each picture shows letter and digital characters and graphic symbols. Usually, changeable colors, graphs and flashing are adopted to indicate different states of process variables. The value and state of all process variables are dynamically refreshed every second.

Operator operates and adjusts related process variables in this screen.

\section{Bar Graphs}

Bar graphs show the variation of process variables. For example, bar graph can be used to indicate the liquid level of tower vertically or horizontally. One screen can display 40-point horizontal or 64-point vertical bar graph.

Report Screen

The report screen displays and inquiries analog signal in the system and information of on/off state change of switch value, including label name, change state, change time, report, and record printing function.

Reporting Function

The CPS system prints reports according to the pre-defined format. The collection and printing of report data can be conducted automatically in the user-defined interval or by means of event driven or by means of operator command.

Report Type:

- Class report

- Daily report

- Monthly report

Record and Record Printing Function

In the case of change of alarm of any type, information, control parameter, control mode and operational attribute, CPS system will immediately start the printer for printing, and generate log database for future inquiry. The log record cannot be changed manually at will.

This system provides a storage space larger than 100G. Hence, the historical data of alarm, information, operational instructions, historical trends and so on can be reserved longer than half a year. Besides, the abovementioned data can be automatically or manually transferred to external storage medium, such as CD, magnetic disk, MO, etc.

System Maintenance Screen

System maintenance personnel can easily maintain the whole system via the system maintenance screen. From this screen, the maintenance personnel can observe the state of the whole system and the location and cause of system failure. The system maintenance screen contains the following contents:

- System diagnosis picture

- System maintenance picture

- System working condition picture

- Equipment status picture

\section{AHC2001 Hybrid Controller}

AHC2001 hybrid controller supports combinations of various types: control loop, logical function, sequential control, and I/O processing. Hence, users can combine the functions they desire most to address their application demands.

I/O Processing

- Input linearization/conversion to engineering unit

- Multistage alarm

- Input diagnosis

- Output restriction and normalization

PV Calculation

- Algorithm (accumulation, add-sub. multi-div. etc.)

- Calculator function

- Multistage alarm 
Control Calculation

- Algorithm (PID-a, ra-PID, up-PID. etc.)

- Initialization and track

- Security

Logical Control and Combination of Digit

- Boole, timer and comparator

- Input diagnosis and chain

- Circuit disconnection alarm and chain

Sequential Control Programming

- Parallel operation

- Embedded exception handling

- Batch order

- Prescription data and intrinsic parameters

\section{System Diagnosis and Fault-tolerant Capability}

CPS system is provided with multiple diagnostic tools, and the diagnosis result can be displayed in multiple modes. Each AHC module is provided with an LED indicator for indicating its state. The contents to be indicated via the LED are:

- Controller module (CPU module)

Power/run/main LED and status display

- Ethernet interface module

Run/power LED

In addition to LED indicator, the CPA also displays the system state, AHC state, and errors and realize confirmation.

CPS is a system containing multiple CPUs, of which each module can independently fulfill its functions. Thus, any module with failure will not affect other modules.

\section{Print Server}

It can be realized to operate the historical database in the plant to automatically collect engineering operation data, and store the collected data as historical data for future use. Trend and status display can be realized via client software (correlation/backspace analysis, historical graphics, etc.), can connection to MS Excel/Access and browser software also can be realized.

\section{System Communication}

For the communication, peer-to-peer digital communication system consisting of shielded twisted pair and switch is adopted, of which the transmission capacity reaches up to 10 Mbaud /100Mbaud. The switch is provided with 10BASE-T and 10BASE5 communication interfaces, which can meet information networking and management functions of a whole plant, and its communication protocol meets IEEE802.4, which can realize communication with equipment supporting TCP/IP protocol of a certain number that can meet the needs of LAN of a whole plant.

\section{Power Supply System}

Retention Time after Power Failure of Main AC Power Supply

Retention time after power failure under normal circumstances or in the case of full load: calculate according to the UPS capacity.

DC24V Power Supply

Except for digital output via electromagnetism value, all required DC24V is provided by the system.

\section{Software}

We will provide advanced, reliable and convenient system software. The software consists of software required by operating system, configuration, download, diagnosis and communication, as below: 
- Control and monitoring software SLPD-50

This software is the latest software providing system configuration, application software download, system diagnosis and system communication and such functions, and also an indispensable software for project control system.

- Application software CPA

Point configuration, logical chain, loop control scheme, sequential order program, image configuration and report, and so on manufactured in accordance with the needs of the buyer and the technological requirements.

The abovementioned softwares are of the latest edition, and we can update software for users for free, provided that the base edition remains unchanged.

\section{System Configuration Description}

\section{Control Program Assist (CPA):}

1. Upper Operation Station:

1 sets

- Pentium 4 2GMHz CPU

- 40 GB HDD

- 512MB memory

- Mouse

- 50X CD RW driver

- QWERTY type 101 keyboard

Among the 2 sets, one is of universal attributes, that' $s$ it supports both operator attributes and engineer attributes; and the other is of operator attributes, which is for operator use only.

2. 19" color monitor:

1 set

- Resolution ratio $1024 * 768$

3. Engineer keyboard:

1 set

4. Concentrator:

5. Operation desk:

1 set

1 nos.

\section{Printer and Printer Bench:}

1. Color ink jet printer * set

Report printing and picture copying

2. Line-at-a-time printer $\quad *$ set

Alarm printing

\section{Controller Cabinet and Distributor}

1. AHC controller cabinet and distributer 1 coil

Both the controller and expander board are installed in the cabinet.

\section{Cables}

1. The company provides cables as below:

- Communication cable (30 meters long for Ethernet network)

- Extension cable: 3 meters long for installing in the cabinet to connect the controller to the expander board.

- Peripheral cable of system (for printer).

2. The required products that the company doesn' t provide include:

- System cables other than the ones listed above

- On-site I/O cable self-provided in the cabinet. 


\section{Schematic Diagram of Control Network Composition}

Distribution Diagram of Upper Monitor System and Controller

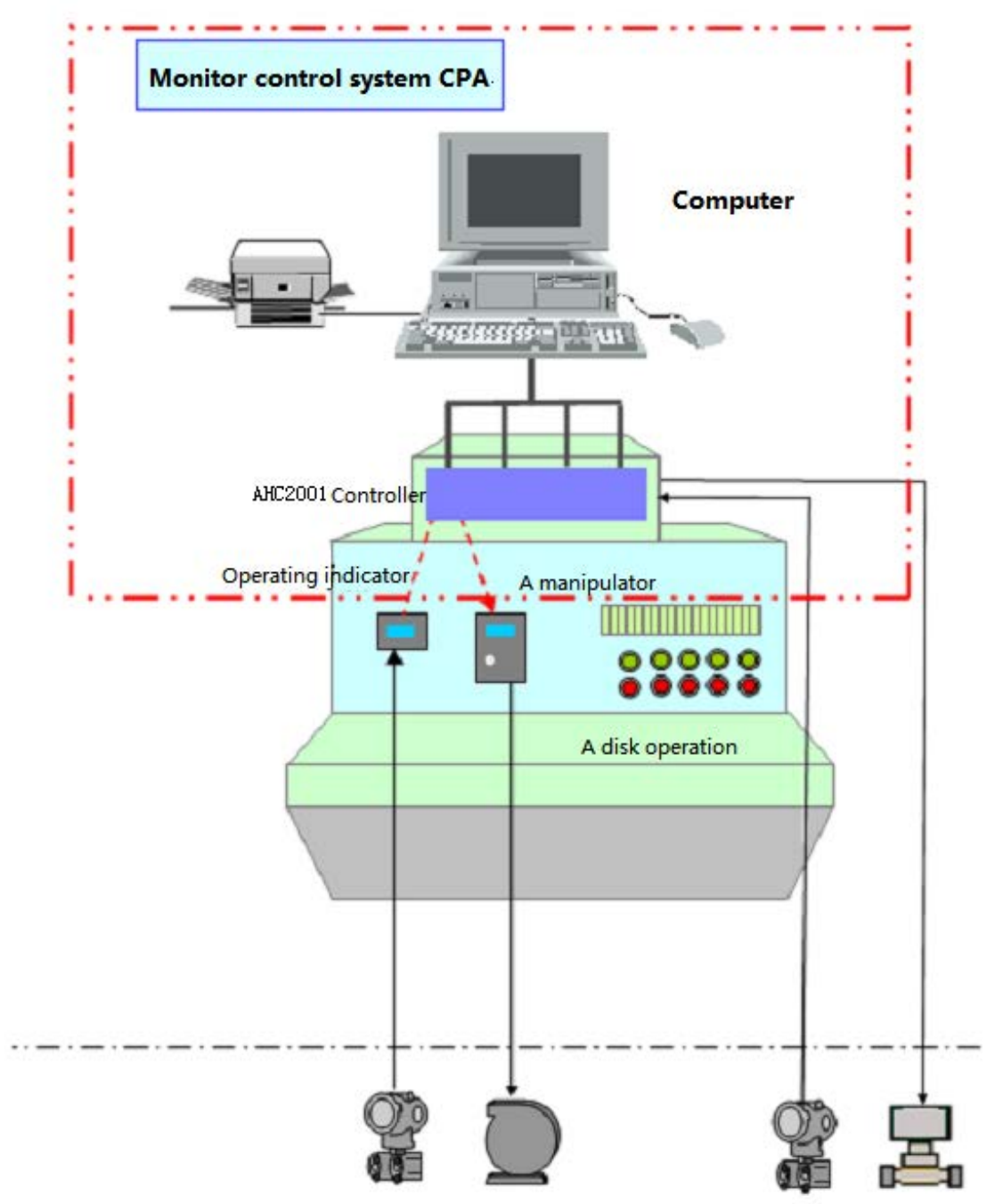

CPS System and Network Structure Diagram 


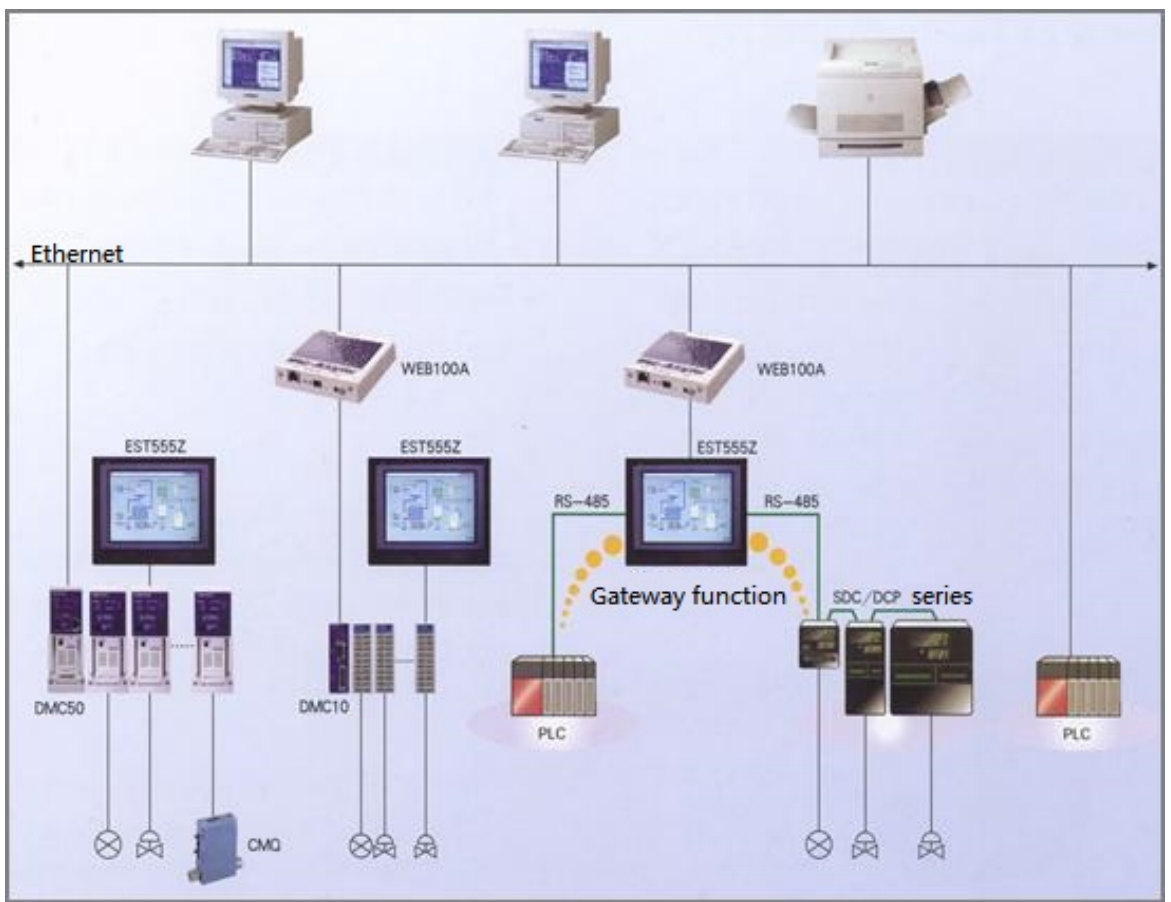

Single Line Diagram of Power Distribution 


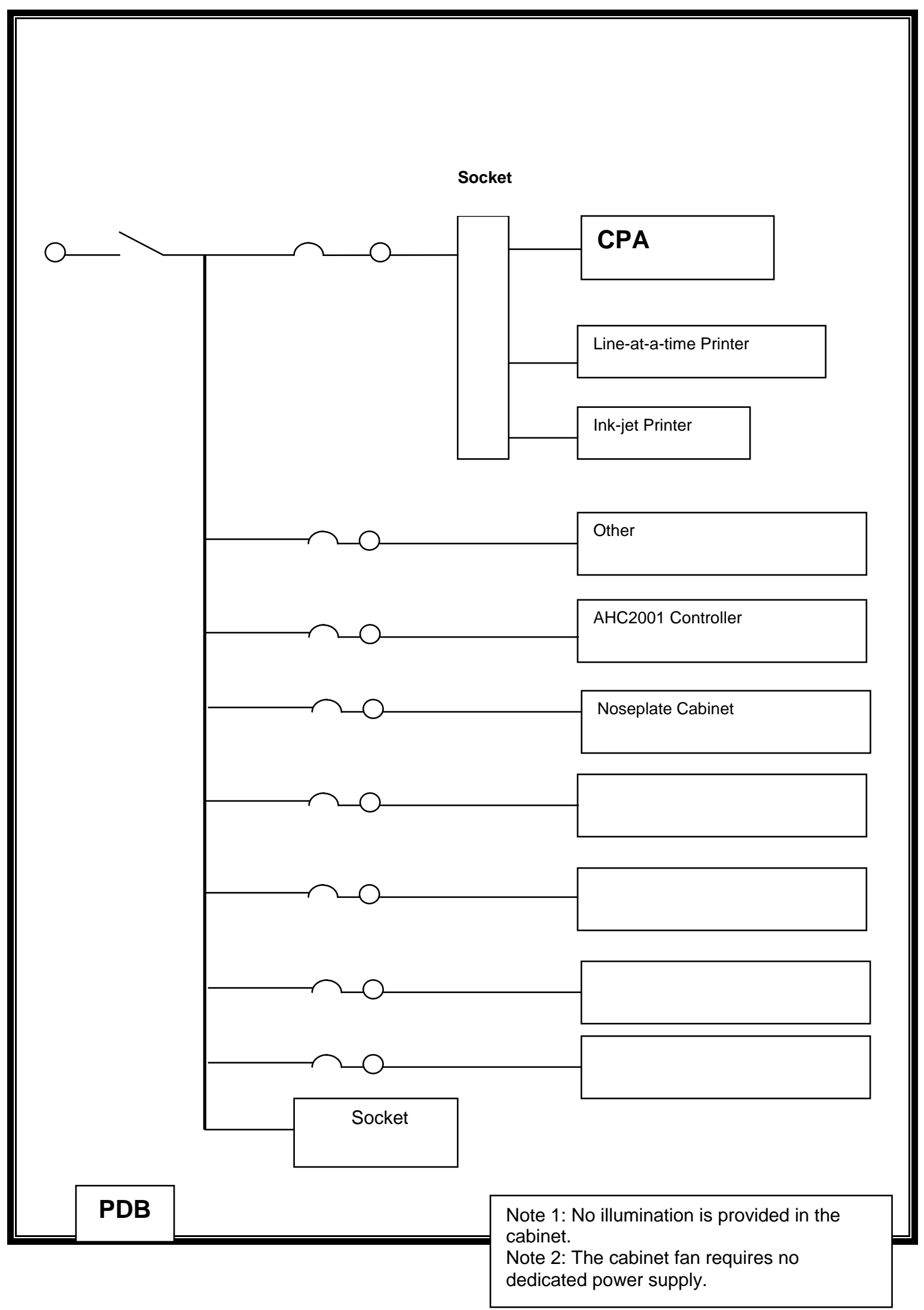

\section{Conclusion}

For the whole set of the control system, we adopt a design idea of decentralized control and centralized management. For the host computer, an industrial personal computer is employed, so that the stability and reliability can be guaranteed in severe environment such as high temperature. Automatic control technology is an important product of the development of the computer age, which 
needs to be backed by strong comprehensive national strength and science and technology capability. This is also the goal of industrial development of our country. Presently, automatic control technology is almost used in all manufacturing, control and chemical enterprises, but we still have to import most hardware, software and systems from Europe, America and Japan. Therefore, cultivating and introducing technical talents have become the only way to improve China' s industrial production level.

\section{References}

[1] Wang Haijun. Evaluation of Reliability of Electric Automatization Control System of Power Plant, Foreign Investment in China, 2012, (19).

[2] Li Jing. Evaluation of Reliability of Electric Automatization Control System of Gangue Power Plant, Energy and Energy Conservation, 2015, (1).

[3] Song Wenkai. Humble Opinions on Application of Electric Automation Technology-Case Study of Huangling Mining Industry Gangue Electricity Generating Co., Ltd., China High-tech Enterprises, 2016, (1).

[4] Li Chunguang. Application of Automation Technology in Electrical System of Power Plant, China High-tech Enterprises, 2014, (2).

[5] Wang Hongying. Discussions on Reliability of Electric Automation System, Science \& Technology Vision, 2014, (4). 